\title{
THE USE OF SODIUM FLUORESCEIN IN ASSESSING THE RATE OF HEALING IN CORNEAL ULCERS*
}

BY

\author{
F. W. Campbell and T. A. S. Boyd \\ Institute of: Physiology, University of Glasgow.
}

For nearly 70 years a solution of sodium fluorescein has been used by ophthalmologists for initial detection of breaks in the continuity of the epithelium of the cornea (Pflüger, 1882; Straub, 1888). In contrast, sodium fluorescein has rarely been used to follow the course of healing in corneal lesions or to determine the time taken for restoration of an epithelial covering over a corneal injury. In view of the need for comparing and assessing the efficacy of an ever increasing number of chemotherapeutic drugs and of antibiotics, a reliable method for following the course of healing of corneal ulcers is urgently required.

\section{Action of Fluorescein}

A solution of sodium fluorescein does not readily penetrate the healthy epithelium of the cornea. If allowed to remain in contact with the normal epithelium for a sufficient length of time, however, fluorescein can penetrate to the underlying substantia propria of the cornea (Fromm and Groenouw, 1891). Here the fluorescein, during its passage to the anterior chamber, fluoresces vividly on exposure to ultra-violet light. If the fluorescein solution remains in contact with the healthy epithelium of the cornea for no longer than one minute, practically no penetration takes place and no fluorescence occurs.

When the epithelium is absent, devitalized or thinner than normal, application of a solution of sodium fluorescein for one minute now leads to penetration, and the underlying stroma fluoresces on exposure to ultra-violet light.

As an ulcer heals, the corneal epithelium spreads over the surface of the ulcer and increases in thickness. Simultaneously the intensity of fluorescence, after application of fluorescein solution under standard conditions, decreases until it finally ceases.

This end-point is, however, obviously dependent on the various arbitrary features of the test. Moreover, the end-point, disappearance of fluorescence, is no guarantee that the reparative processes are all complete. Indeed, Campbell, Ferguson, and Garry (1950) showed in guinea-pigs that, after injury to the cornea, weakness of the stroma persisted for several weeks after cessation of fluorescence.

\footnotetext{
* Received for publication May 5, 1950.
} 
Nevertheless, if a standard technique in the use of sodium fluorescein is rigidly adhered to, it should be possible to gauge with some accuracy the course of healing of corneal lesions. The end-point where fluorescence ceases makes it possible to evaluate, statistically if necesary, the efficacy of various remedial measures.

To follow the regression in intensity of fluorescence and consequently the course of healing, it is necessary to establish standards of reference which can be used to estimate the progress in healing at each examination.

\section{Preparation of Standards}

The preparation of suitable standards of reference required an investigation into the relationship of the concentration of sodium fluorescein to its intensity of fluorescence.

We impregnated strips of Whatman No. 1 filter paper with known quantities of sodium fluorescein (B.D.H.). The sodium fluorescein was dissolved in 50 per cent. alcohol, and a known volume of this solution evenly distributed on a piece of filter-paper of known area. This strength of alcohol was found to dry more rapidly than a watery solution and to allow more even distribution of the stain. Higher concentrations of alcohol were found to be unsuitable because sodium fluorescein was incompletely dissolved. The rapid drying necessary for even distribution was ensured by holding the paper in a stream of hot air from a hair dryer.

We prepared a number of strips with concentrations of sodium fluorescein ranging from 0.125 to $128 \mathrm{microg} . / \mathrm{sq} . \mathrm{cm}$. of filter

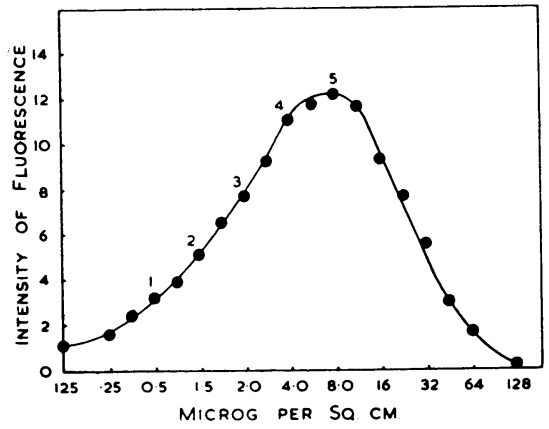

FiG. 1.-Relation of intensity of fluorescence (galvanometer readings) to concentration of fluorescein in microg./sq. $\mathrm{cm}$. of filter paper (logarithmic scale). The points 1 to 5 on the graph correspond to the concentrations chosen for clinical use. paper. These strips were exposed to ultra-violet light from a G.E.C. mercury vapour ultra-violet dark bulb lamp. The intensity of the fluorescence was then measured photometrically with a low resistance galvanometer and a barrier-layer photoelectric cell. To absorb ultraviolet light the photocell was screened with a green $W$ ratten filter $(61 \mathrm{M})$. The impregnated strip of filter paper was placed in direct contact with this filter. The relative intensities of the light emitted from the fluorescein- 
impregnated paper was then read directly on the galvanometer scale. This photometer gave a direct linear relationship between the fluorescent light intensity and the galvanometer reading.

Fig. 1 shows graphically the results obtained. For convenience a logarithmic scale was used on the abscissa. The graph shows clearly that the intensity of the fluorescence rises to a maximum with increasing concentration of fluorescein up to $8 \mathrm{microg}$./sq. $\mathrm{cm}$. Beyond this point, increase of concentration of fluorescein not only fails to increase the fluorescent response on exposure to ultra-violet light, but actually diminishes it. This phenomenon, well known to physicists, is called "quenching "; the explanation is obscure (Pringsheim, 1949).

From inspection it may be seen that concentrations of fluorescein ranging from 0.5 to 8 microg./sq. $\mathrm{cm}$. give a spread of intensity most useful in the preparation of standards for clinical use. Over this range a geometric increase in the concentration of fluorescein gives an almost linear increase in intensity of fluorescence. Moreover, the hue of the fluorescent light emitted is very similar to that from corneal lesions. Greater concentrations are not suitable

TABLE

Relation of the Concentration of Sodium Fluorescein to the Intensity of Fluorescence from the Standard Strips of Filter Paper for Clinical Use.

\begin{tabular}{c|c}
\hline $\begin{array}{c}\text { Intensity of } \\
\text { Fluorescence }\end{array}$ & $\begin{array}{l}\text { Concentration of } \\
\text { fluorescein in } \\
\text { microg./sq. cm. } \\
\text { of filter paper }\end{array}$ \\
\hline 0 & \begin{tabular}{c} 
No fluorescein \\
\hline 1
\end{tabular} \\
\hline 3 & 0.5 \\
\hline 4 & 2 \\
\hline 5
\end{tabular}

for clinical standards because the deep orange colour of the concentrated fluorescein interferes with the hue of the fluorescence.

For clinical use, five standard filter paper strips were chosen with concentrations in the range of 0.5 to 8 microg. sodium fluorescein $/ \mathrm{sq}$. cm. From the graph it can be seen that the intensities of fluorescence emitted from these strips bear, for all practical purposes, a linear relationship to each other. For simplicity we chose to represent these intensities by the numerals $1,2,3,4$, and 5 (see Table). These numerals are approximately proportional to the absolute intensity of fluorescence. Since this absolute intensity depends also on such factors as the distance and strength of the light source, standardization of working conditions is essential. 
For convenience we mounted the five strips behind glass under a black paper mask which exposed an area of $1 \times 0.5$ in. of each strip. There has been only slight fading over a period of nine months.

\section{Use of Impregnated Strips to assess the Course of Healing}

The clinical use of a method such as this must be carefully standardized. One drop of a 2 per cent. aqueous solution of sodium fluorescein is instilled into the conjunctival sac. After exactly one minute the eye is washed with enough isotonic saline to remove excess fluorescein solution. The set of standard strips is then held beside the patient's eye.

Comparison is made under illumination by a G.E.C. ultra-violet lamp placed at a convenient distance, say two feet. The intensity of fluorescence from the lesion is then matched against the strips.

Ideally the matching should be carried out in darkness, but is also satisfactory if the surrounding illumination is constant and of moderate intensity.

Using a similar method, Campbell and others (1950) were able to follow the course of healing in deep corneal burns in guineapigs, and to show that there was marked delay in scorbutic animals.

The method was equally successful in assessing the value of

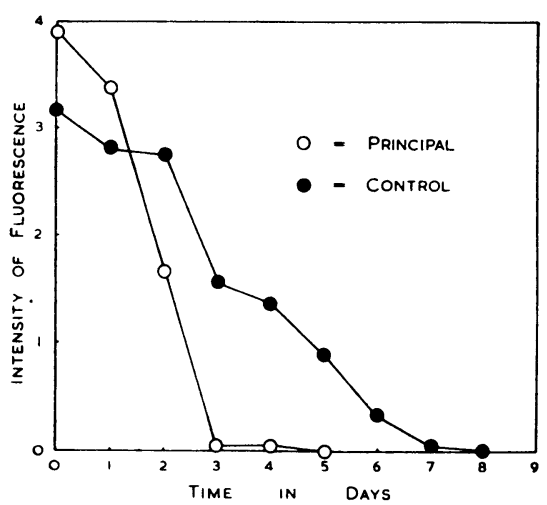

FIG. 2.-Mean intensity of fluorescence of corneal ulcers treated with (principals) and without (controls) ascorbic acid plotted for each day during healing. The figures on the ordinate correspond to the strip intensities described in the text. massive doses of ascorbic acid in the treatment of corneal ulceration in human beings (Boyd and Campbell, 1950). Fig. : (also reproduced in that paper) indicates that this method of observation has demonstrated a marked difference in the rates of healing of patients who had and had not received ascorbic acid. It has also given a clear indication of the effect of the ascorbic acid as early as the second day of treatment.

Furthermore, the method sharply detected the precise time of cessation of fluorescence in each ulcer. This procedure facilitates statistical comparison of two or more groups of patients undergoing different forms of therapy. 
We wish to thank Doctors J. Pendleton White, J. Hill, A. Wright Thomson and A. Mellick of the Glasgow Eye Infirmary, for permission and facilities to examine their patients, upon whom the method outlined in this paper was used.

We also wish to express our gratitude to Professor R. C. Garry and to Dr.

J. B. de V. Weir for their kindly criticism and encouragement.

We are indebted to the Rankin Medical Research Fund of the University of Glasgow for defraying the cost of this investigation.

\section{Summary}

A clinical method is described for assessing the rate of healing of corneal ulcerations and injuries. The end-point of epithelization can be accurately determined.

\section{REFERENCES}

Boyd, T. A. S., and CAMPBELL, F. W. (1950): Brit. med. J. In the press.

Campbei.l, F. W., Ferguson, I. D., and Garry, R. C. (1950). Brit.J. Nutrit. In the press.

Fromm, and Groenouw (1891). Arch. Augenheilk., 22, 247.

Pfil̈ger (1882). Klin. Mbl. Augenheilk., 20, 69.

Pringsheim, P. (1949). “Fluorescence and Phosphorescence”. Interscience Publ., New York.

Straub, M. (1888). Zbl. prakt. Augenheilk., 12, 75. 